\title{
The Blind Location Tracking System Based on ARM
}

\author{
Liqun $\mathrm{Bao}^{1}$, Zhiwei $\mathrm{Hou}^{2}$, Zhongdong $\mathrm{Wu}^{2}$, Xianglin $\mathrm{Li}^{1}$ \\ ${ }^{1}$ School of Electronic \& Information Engineering, Lanzhou institute of technology, Gansu Province, China \\ ${ }^{2}$ School of Electronic \& Information Engineering, Lanzhou Jiaotong University, Gansu Province, China \\ 'baoliqun1983@163.com
}

\begin{abstract}
The purpose of this paper is to introduce a blind location tracking system which can be used by the family to keep track of the blind. The whole system consists of three modules, ARM development board, $3 \mathrm{G}$ wireless communication module and a smart phone. The important steps in this study is to transfer the Android operating system into ARM development board, get the blind's location information and display it on the screen of the ARM development board on the basis of the Android platform using Google Maps. The blind take smart phones with them when they go out. Their family can see their location in real-time through the system. In case of any emergency, the blind can get help in time, increasing the safety of travel for the blind, also reducing the concerns of the family.
\end{abstract} Maps

Index Terms - The blind location tracking, ARM, GPS, Google

\section{Introduction}

According to the latest figures released by the world health organization, about 39 million people around the world are blind. There are 246 million people have moderate or severe visual impairment, it is a huge social group. This organisation predicted that with the growth of population and the aging trend, if we do not take effective measures, the total number of the blind man and people with vision problems in the world will be doubled in 2020 [1].

Along with the progress of the society, people increasingly pay attention to the harmonious development of human society, and the concern on the blind and other disadvantaged groups is increasing. In order to facilitate the travel of the blind, Guangdong Mobile Communication Corporation developed mobile positioning system for the blind. The blind can query their location in the city of Guangzhou from the 96931 mobile phone service[2]. Early research focused primarily on seeing-eye products both at home and abroad which have the function of seeing-eye, obstacle avoidance, location awareness, etc. However, some hidden safe troubles still exist, such as inaccurate detection and low sensitivity. Due to busy work and other reasons, the family can not always accompany the blind. They hope to be able to to keep track of the blind in real-time, to ensure the safety of the blind.

The paper implements the blind location tracking system based on ARM. The blind take smart phones with them when they go out. Their family can see their location on the screen of ARM development board in real-time. In case of any emergency, the blind can get help in time, increasing the safety of travel for the blind, also reducing the concerns of the family.

\section{System Architecture and Workflow}

As shown in figure 1 , the blind location tracking system consists of a smart phone carried by the blind, $3 \mathrm{G}$ wireless communication module and ARM development board. There are three steps of the design as follows:

1) Configure and compile the Android kernel (Linux 2.6.35.7), then transfer the Android operating system into ARM development board.

2) Get the blind's location information (longitude and latitude)on the smartphone through GPS positioning or wireless communication network[3], then the blind location information is sent to the development board periodically through the $3 \mathrm{G}$ wireless network .

3) Complete the blind location tracking system and display the location of the blind on the screen of the ARM development board on the basis of the Android platform using Google Maps.

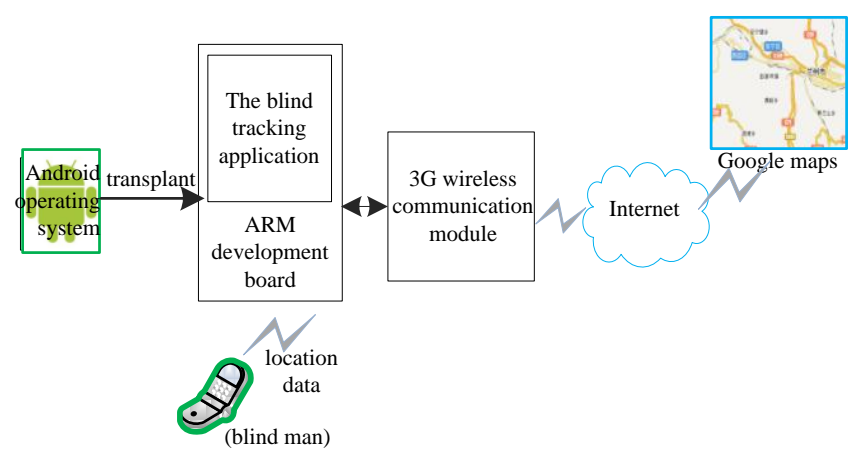

Figure 1. System architecture

\section{The Implementation of Key Technology}

\section{A. The porting of Android operating system into the ARM platform}

Tiny 210 is a high-performance Cortex-A8 core board, using the samsung S5PV210 as the main processor, it's runtime frequency can reach $1 \mathrm{GHZ}$. The S5PV210 integrates PowerVR SGX540 high performance graphics engine, it can run operating systems such as Android, Linux and WinCE6 etc, suitable for the development of high-end monitoring system and advertising multimedia terminals. It has the rich I/O pins and interface resources, can conveniently extend the functional modules.

1) Cutting and compilation of the Linux kernel

The Linux kernel that is used by Android is different from standard Linux. The following are the steps of the 
configuration.

\#cp mini210_linux_defconfig .config ;

\#make menuconfig ARCH=arm $/ *$ make configuration $* /$ \#make /* to compile*/

2) Making file system image

Using mkyaffs2image-128M tool, make the directories of target file system into yaffs 2 -format image file, then write it to Nand Flash, and start.The entire root directory becomes yaffs 2 file system format, the default Android kernel has already supported this file system. Enter the following command at the command line:

\#mkyaffs2image-128M rootfs_dir rootfs_android.img

3) The installation of the cross-compiler

The paper uses arm-Linux-GCC-4.5.1, its installation steps are as follows:

\#tar xvzf arm-linux-gcc-4.5.1-v6-vfp-20101103.tgz -C /

\#gedit/root/.bashrc

Edit /root/bashrc file, amend the last line:

export

PATH=\$PATH:/opt/FriendlyARM/toolschain/4.5.1/bin

\section{B. Getting GPS location data}

NMEA0183 protocol is the GPS interface protocol standard established by the American National Marine Electronics Association[4]. NMEA0183 defines a number of sentences of different meanings, each statement is actually an ASCII string. A complete NEMA0183 statement starts at operator "\$GPGGA", and end with terminator "< CR $><$ $\mathrm{LF}>"$. The information we need to get is the latitude and longitude, and their direction, GPS positioning state and reception time. So when it receives a complete NEMA0183 statement, the method of extracting useful information is: first find the position of the starting character "\$GPGGA", read in data from the starting character, then look for characters ', ' after xor check, finally capture the character or character string between two nearby", to get useful data, and use carriage return as a termination character of GPS statement. After getting a complete GPS signal. Find the latitude and longitude coordinates in GPS signal and lead them out, and then convert to degree.

\section{The Realization of Location Tracking System}

\section{1) Technical principles}

Google Maps is an electronic map service provided by Google. Before using the Google Map service in Android applications, applying for a Google Map Android API Key is necessary[5]. ARM development board is connected to the internet by $3 \mathrm{G}$ wireless communication module, and then access the Google maps application server. Call the class libraries of related position location provided by the Android platform and the GoogleMaps, to realize the the conversion between the blind's address and geographic coordinates, then the geographical coordinates are converted to the corresponding mobile phone pixels. In the development of the application of Google maps, represent any place in the earth through the combination of the longitude and latitude. Class Point (com.google.android.maps.Point) represents a latitude/longitude point.
2)The realization of the positioning function

The background process is responsible for the monitoring of the User's location changes. Surveillance behavior is implemented by the LocationListener interface. If there are changes, the system will update the user's location in the network map. The construction method of latitude and longitude point is as follows [6]:

GeoPoint (int latitudeE6, int longitudeE6)//Constructs a GeoPoint with the given latitude and longitude, measured in microdegrees (degrees * 1E6).

The following code implements positioning to the fourth teaching building of Lanzhou Institute of Technology, which

lies at $103^{\circ} 43^{\prime}$ east longitude, $36^{\circ} 5$ 'north latitude.

public void onCreate(Bundle savedInstanceState) \{

super.onCreate(savedInstanceState);

setContentView(R.layout.main);

this.mapView $=($ MapView $)$

this.findViewById(R.id.map_view);

this.mapView.setBuiltInZoomControls(true);

GeoPoint point=new GeoPoint(36050000, 103430000);

this.mapController=mapView.getController();

this.mapController.animateTo(point); \}

\section{System Test Result}

We test the function of this system on the Tiny 210 development board based on CortexTM-A8, Andriod 2.3 operating system and Samsung's smartphone. Figure 2 is the physical map for the blind Location tracking system. Sumsung smart phones is on the left side of Tiny 210 development board.

The blind take smart phones with GPS chip with them when they go out. Their family can see their location on the screen of ARM development board in real-time. As shown in figure 3 , the system displays the position of the blind who is in the Lanzhou city, Qilihe District, Gongjiawan crossing, about 8 meters away from the China Construction Bank.

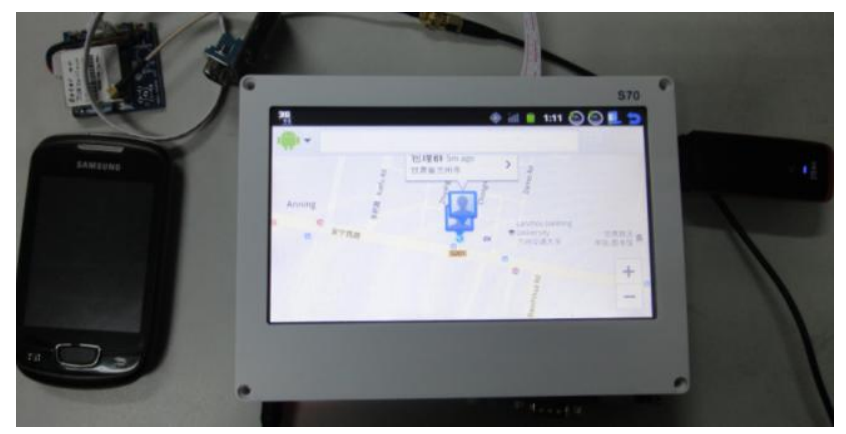

Figure 2. The physical map of the blind location tracking system 


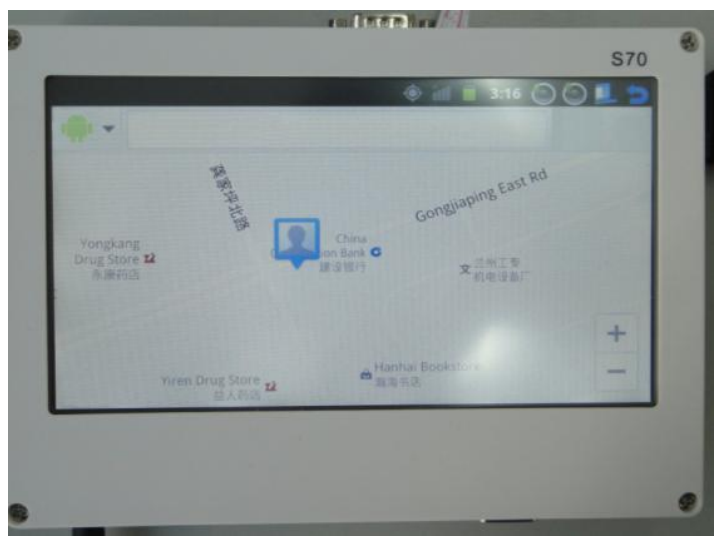

Figure 3. The location map of the blind showed by system

\section{Conclusion}

This blind location tracking system has a good environmental adaptability. It can be used in $3 \mathrm{G}$ network coverage area. It is suitable for the blind and low vision group, also applies to the groups that need special care such as the elderly and children. The user interface is friendly and each module works well. The power consumption is low and the positioning accuracy is within 10 meters. We hope to extend the function of the system by adding some other features such as emergency alarm.

\section{Acknowledgements}

The research is supported by Higher school scientific research project of Gansu Province (Grant No.2013A-127), Gansu Provincial Department of Education scientific research project (Grant No.20971), Gansu Provincial science and technology support program (Grant No. 1104GKCA032).

\section{References}

[1] World Sight Day, (October, 2011), available: http://news.xinhuanet. com/ziliao/2008-10/08/content_10164460. htm.

[2] Guangzhou blind people use mobile positioning system, (May, 2005), available:http://news.sohu.com/20050528/n225736955.shtml.2005.5.28.

[3] Yuan YuanYuan, Zhang LiJun. "Design and Implementation of Positioning and Navigation System Based on Android", National Conference on Information Technology and Computer Science (CITCS 2012), pp.686-689.

[4] QIAN De-jun, ZHANG Zhe, HU Chen, On Parsing of NMEA0183 Protocol, Chinese Journal Of Electron Devices, 2007,30(2).

[5] Google Maps Android API, available: https://developers.google.com/maps/documentation/android/index/.

[6] Gao Cai-li,Xu Li-ming,Yuan Hai.Android application development paradigm precise explanation. Tsinghua University Press, Beijing, 2012. 